\title{
Maximal cuts and Wick Rotations
}

\section{Ettore Remiddi ${ }^{a, *}$}

${ }^{a}$ DIFA-INFN,

Via Irnerio 46, Bologna, Italy

E-mail: ettore.remiddi@bo.infn.it

The evaluation of a maxcut amplitude by the direct integration of the loop momenta gives in general a vanishing result, as the $\delta$ functions impose overconstrained restrictions to the integration region. It is proposed to relax the constraints, so that a non vanishing result for the maxcuts can be obtained, by giving Minkoskian (rather than Euclidean) metric to the components not spanned by the physical momenta, including the regularising components of the continuous regularisation scheme. As an example, the one and two loop Bhabha box amplitudes are considered.

MathemAmplitudes 2019: Intersection Theory \& Feynman Integrals (MA2019)

18-20 December 2019

Padova, Italy

\footnotetext{
${ }^{*}$ Speaker
} 


\section{The maxcut amplitudes}

If a Feynman propagator is written as

$$
\frac{-i}{D-i \epsilon}=\mathcal{P}\left(\frac{-i}{D}\right)+\pi \delta(D),
$$

the corresponding cut propagator is

$$
\delta(D)
$$

if a propagator is squared

$$
\frac{-i}{(D-i \epsilon)^{2}}=-\frac{d}{d D}\left(\frac{-i}{D-i \epsilon}\right),
$$

the corresponding cut squared propagator is

$$
-\delta^{\prime}(D)=-\frac{d}{d D} \delta(D),
$$

and so on for higher powers and derivatives.

Given any Feynman graph amplitude the corresponding maximally cut amplitude (or maxcut amplitude, or even maxcut for short) is obtained by replacing all the propagators of the amplitude by the corresponding cut propagators defined by the above rules.

If a Feynman amplitude satisfies an integration by part (or $i b p$ ) identity, the corresponding maxcut identity satisfies the same identity, with all the propagators replaced by the corresponding cut propagators.

An important remark is however in order: if the obvious simplification

$$
D \frac{-i}{D-i \epsilon}=-i
$$

applies to a term occurring in the $i b p$ Feynman amplitude with the first power of $-i /(D-i \epsilon)$ only (i.e. with a simple propagator, not a squared one), the simplification generates a term without the considered propagator (usually called an inhomogeneous term) in the identity. In the corresponding identity for the maxcut amplitude the simplification reads

$$
D \delta(D)=0,
$$

so that the corresponding inhomogeneous term is missing. Note for completeness that if a term with a squared denominator appears in the Feynman amplitude and the (homogeneous) simplifications

$$
D \frac{-i}{(D-i \epsilon)^{2}}=\frac{-i}{D-i \epsilon}
$$

occurs, the corresponding simplification in the cut amplitude reads

$$
D\left(-\delta^{\prime}(D)\right)=\delta(D),
$$

so that the simplified (homogeneous) term is present also in the maxcut amplitude.

The differential equations for the Master Integrals of the Feynman amplitude, which follow at once from the inhomogeneous ibp identities, are inhomogeneous as well, and are usually solved (Euler's 
method) by trying to solve the corresponding homogeneous equations as a first step.

From the $i b p$ identities for the maxcut amplitudes one can also obtain at once the differential equations for the maxcut Master Integrals; those equations are obviously homogeneous given the absence of inhomogeneous terms in the $i b p$ identities for the maxcut amplitudes, and are exactly the homogeneous part of the inhomogeneous system obtained for the Feynman amplitudes.

As a result, the maxcut amplitudes are solutions of the homogeneous equations valid for the Feynman amplitudes, so that the direct evaluation of maxcut amplitudes (surely simpler than the direct evaluation of the corresponding Feynman amplitudes) provides with a powerful tool for the solution of the differential equations.

In particularly simple cases (including however also the non trivial massive sunrise graph) the maxcut amplitudes are strictly related to the unitarity cuts, whose direct evaluation was indeed used for obtaining the solutions of the homogeneous part of the differential equations. But in more general cases, the direct evaluation of the maxcut amplitudes as an integral on the components of the loop momentum gives a vanishing result, as it involves too many $\delta$-function constraints for too few integration variables and does not provide information on the solution of the homogeneous equations (which admit always a vanishing solution).

In the next paragraphs after a short discussion of the problem, we show that a Wick rotation of some loop momenta can give non vanishing and usful results.

\section{The 1-loop Bhabha box}

As an example, consider the 1-loop Bhabha box

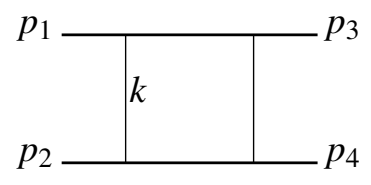

Figure 1: The 1-loop Bhabha box.

The kinematics of the process is $p_{1}+p_{2} \rightarrow p_{3}+p_{4}$, with

$$
\begin{aligned}
p_{1}^{2} & =p_{2}^{2}=p_{3}^{2}=p_{4}^{2}=-m^{2}, \\
p_{1} & =(E, p, 0,0), \\
p_{2} & =(E,-p, 0,0), \\
p_{3} & =\left(E, p_{x}, p_{y}, 0\right) . \\
s & =-\left(p_{1}+p_{2}\right)^{2}=4 E^{2}=4\left(p^{2}+m^{2}\right), \\
t & =-\left(p_{1}-p_{3}\right)^{2}=-2 p\left(p-p_{x}\right), \\
s & +t-4 m^{2}=2 p\left(p+p_{x}\right),
\end{aligned}
$$

and the maxcut, in the (usual) $d$-continuous dimensions, can be written as

$$
B(s, t)=\int \mathcal{D}^{d} k \delta\left(D_{1}\right) \delta\left(D_{2}\right) \delta\left(D_{3}\right) \delta\left(D_{4}\right),
$$


(the overall normalization is irrelevant when looking for solutions of homogeneous equations), with the loop momentum $k$ of components $k_{0}, k_{x}, k_{y}$ and $\vec{K}$, where the (Euclidean) vector $\vec{K}$ has $(d-3)$ regularising components, not coupled to the four external physical vectors $p_{i}$. By using spherical coordinates for $\vec{K}$ one can write

$$
\begin{aligned}
\mathcal{D}^{d} k & =d k_{0} d k_{x} d k_{y} d \vec{K} \\
& =d k_{0} d k_{x} d k_{y} \Omega(d-3) K^{(d-4)} d K
\end{aligned}
$$

where $\Omega(n)=2 \pi^{\frac{n}{2}} / \Gamma\left(\frac{n}{2}\right)$ is the usual solid angle in $n$ dimensions, and

$$
\begin{aligned}
& D_{1}=k^{2}, \\
& D_{2}=\left(p_{1}+k\right)^{2}+m^{2}=+2 p_{1} k+k^{2}, \\
& D_{3}=\left(p_{2}-k\right)^{2}+m^{2}=-2 p_{2} k+k^{2}, \\
& D_{4}=\left(p_{1}-p_{3}+k\right)^{2}=-t+2 p_{1} k-2 p_{3} k+k^{2} .
\end{aligned}
$$

Due to Eq.(2.2), Eq.(2.1) is a (rather simple) integral on four integration variables $k_{0}, k_{x}, k_{y}$ and $K$ of the product of four $\delta$ functions.

Quite in general ( for $i \neq j$ !) one has

$$
\delta\left(D_{i}\right) \delta\left(D_{j}\right)=\delta\left(D_{i}\right) \delta\left(D_{j}+a D_{i}\right)
$$

where $a$ is any real quantity; as an example, with the above $D_{i}$ one can write

$$
\delta\left(D_{1}\right) \delta\left(D_{2}\right)=\delta\left(D_{1}\right) \delta\left(D_{2}-D_{1}\right)=\delta\left(D_{1}\right) \delta\left(2 p_{1} k\right)=\delta\left(D_{1}\right) \delta\left(D_{2}^{\prime}\right)
$$

which amounts to the replacement $\delta\left(D_{2}\right) \rightarrow \delta\left(D_{2}^{\prime}\right)$, with $D_{2}^{\prime}=D_{2}-D_{1}=2 p_{1} k$. By repeated use of the replacements allowed by Eq.(2.4), one arrives at

$$
B(s, t)=\int d k_{0} d k_{x} d k_{y} \Omega(d-3) \int_{0}^{\infty} K^{d-4} d K \delta\left(D_{1}^{\prime}\right) \delta\left(D_{2}^{\prime}\right) \delta\left(D_{3}^{\prime}\right) \delta\left(D_{4}^{\prime}\right),
$$

where the new arguments of the $\delta$ 's, written in terms of the integration variables, are

$$
\begin{aligned}
& D_{1}^{\prime}=k_{y}^{2}+K^{2}, \\
& D_{2}^{\prime}=4 p k_{x}, \\
& D_{3}^{\prime}=2 E k_{0}, \\
& D_{4}^{\prime}=-t-2 p_{y} k_{y} .
\end{aligned}
$$

It is apparent that $D_{1}^{\prime}$ cannot vanish, so that $\delta\left(D_{1}^{\prime}\right)=0$ and therefore

$$
B(s, t)=0
$$

$B(s, t)=0$ is indeed a solution of the homogeneous equation for the Bhabha amplitude, but obviously of no interest.

To get a non trivial result, one can give a Minkowski metric to the $(d-3)$ regularising components of $\mathcal{D}^{d} k$. That is immediately achieved by a Wick rotation (or perhaps counter-rotation), which 
gives an overall factor $(-i)^{(d-3)}$ (irrelevant for the maxcut, as the maxcut is required to satisfy a homogeneous equation). As already observed, those regularising components do not mix with the physical external vectors (and therefore the Wick rotation, when the factors $i$ are included, does not change the value of the original Feynman amplitude); the only effect of the change of the metric is to modify $D_{1}^{\prime}$ :

$$
D_{1}^{\prime}=k_{y}^{2}+K^{2} \rightarrow D_{1}^{\prime}=k_{y}^{2}-K^{2} .
$$

The integrations are now trivial and the result is

$$
B(s, t)=\frac{1}{4} \Omega(d-3)\left[\frac{-t\left(s-4 m^{2}\right)}{4\left(s+t-4 m^{2}\right)}\right]^{\frac{d-4}{2}}\left(-\frac{1}{t \sqrt{s\left(s-4 m^{2}\right)}}\right),
$$

which is positive, as expected ( $t$ is spacelike and therefore negative).

A short comment might be appropriate here. As already recalled, any propagator can be written as

$$
\frac{-i}{D-i \epsilon}=\mathcal{P}\left(\frac{-i}{D}\right)+\pi \delta(D)
$$

when that is done, the product of the the 4 propagators of the 1-loop Bhabha box, Fig.(1), becomes the sum of 16 terms, the product of the $4 \delta$ 's being just one of those 16 terms.

The amplitude, which is the sum of the 16 teerms, does not change, apart from the overall factor $(-i)^{(d-3)}$, if the $(d-3)$ regularising dimensions are properly Wick-rotated, but the values of the single terms can change (and $d o$ change). In particular, the product of the $4 \delta$ 's vanishes with the usual Euclidean regularisation but gives the above non vanishing result when the Minkowskian regularising variables are used.

\section{The 2-loop Bhabha double box}

Let us consider now the scalar 2-loop Bhabha double box amplitude of Fig(2), where the orizontal lines from $p_{1}$ to $p_{3}$ and from $p_{2}$ to $p_{4}$ are the massive electron lines, while the vertical lines are the massless photon propagators.

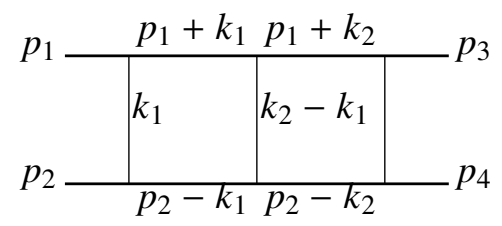

Figure 2: The 2-loop Bhabha double box.

The amplitudes of the 7-propagator graph and of all its subgraphs have been already evaluated analytically in [1], so that, strictly speaking, the knowledge of a single maxcut adds only some more or less marginal details to their knowledge; the hope is that an elementary approach to the planar maxcut, when successfull, might be of help for the treatment of the non-planar Bhabha graph and other similar amplitudes.

The planar double-box Bhabha graph has (of course) the same external kinematics of the 1-loop single-box graphs - but two loop momenta, $k_{1}$ and $k_{2}$ are present. In obvious analogy with the 1loop case, the components of the loop momenta can be taken to be $k_{i 0}, k_{i x}, k_{i y}$ and $\vec{K}_{i}$, with $i=1,2$. 
The scalar products with any the external vectors $p_{i}$, which reads $p_{i} k_{j}=-p_{i 0} k_{j 0}+p_{i x} k_{j x}+p_{i y} k_{j y}$, with $i=1, . ., 4, j=1,2$, do not involve the components of the regularising (Minkoskian) vectors $\vec{K}_{i}$, which enter however into the scalar product of the two loop momenta

$$
k_{1} k_{2}=-k_{10} k_{20}+k_{1 x} k_{2 x}+k_{1 y} k_{2 y}-\vec{K}_{1} \vec{K}_{2} .
$$

It can be convenient to take the direction of $\vec{K}_{2}$ as $z$-axis of $\vec{K}_{1}$, redefining the components of $k_{1}$ as

$$
k_{10}, k_{1 x}, k_{1 y}, K_{1 z}, \vec{K}_{1} \text {, }
$$

where now $\vec{K}_{1}$ has $(d-4)$ components, so that we can write

$$
\begin{aligned}
k_{1}^{2} & =-k_{10}^{2}+k_{1 x}^{2}+k_{1 y}^{2}-K_{1 z}^{2}-K_{1}^{2}, \\
k_{2}^{2} & =-k_{20}^{2}+k_{2 x}^{2}+k_{2 y}^{2}-K_{2}^{2}, \\
k_{1} k_{2} & =-k_{10} k_{20}+k_{1 x} k_{2 x}+k_{1 y} k_{2 y}-K_{2} K_{1 z},
\end{aligned}
$$

and

$$
\begin{aligned}
& \int \mathcal{D}^{d} k_{1}=\int d k_{10} d k_{1 x} d k_{1 y} d K_{1 z} \Omega(d-4) \int_{0}^{\infty} K_{1}^{d-5} d K_{1}, \\
& \int \mathcal{D}^{d} k_{2}=\int d k_{20} d k_{2 x} d k_{2 y} \Omega(d-3) \int_{0}^{\infty} K_{2}^{d-4} d K_{2} .
\end{aligned}
$$

Note that the parametrization of the two loop integration region requires overall 9 variables, while the one loop case requires only 4 variables.

At this point, the calculation of the double box maxcut becomes the (multidimensional) integration of the product of $7 \delta$-functions (corresponding to the 7 propagators) in 9 integration variables, namely the 5 integration variables corresponding to the loop $k_{1}$ and the 4 variables corresponding to $k_{2}$.

The arguments of the $\delta$-functions can be strongly simplified following the procedure leading from Eq.s(2.3) to Eq.s(2.5), and all the integrations are essentially elementary, provided that

- they are carefully performed in a proper order;

- the positivity constraints are also properly accounted for.

As a first step, it is convenient to integrate first the $4 \delta$-functions depending on $k_{1}$ on the 5 variables related to $k_{1}$. The result can be seen as a generalisation of the 1-loop Bhabha box Eq.(2.6) (the four electron lines "external" to the $k_{1}$ loop are all on the mass-shell), but with the would-be (spacelike) Mandelstam variable $t$ replaced by $\left(-k_{2}^{2}\right)$, where the loop-momentum $k_{2}$ can be also timelike.

Indicating by $B_{4}\left(k_{2}\right)$ the itegral of the $4 \delta$-functions of the $k_{1}$ loop, one finds

$$
\begin{aligned}
B_{4}\left(k_{2}\right)= & 2^{-d} \Omega(d-3) \frac{1}{E p}\left(k_{2}^{4}\right)^{-\frac{1}{2}+\frac{d-4}{2}} \\
\times & {\left[\theta\left(k_{2 y}^{2}-K_{2}^{2}\right)\left(k_{2 y}^{2}-K_{2}^{2}\right)^{-\frac{d-4}{2}}\right.} \\
& \left.+\theta\left(K_{2}^{2}-k_{2 y}^{2}\right) \frac{1}{\cos \left(\pi \frac{d-4}{2}\right)}\left(K_{2}^{2}-k_{2 y}^{2}\right)^{-\frac{d-4}{2}}\right] ;
\end{aligned}
$$


due to the constraints of the $\delta$-functions present in the maxcut one has $k_{2}^{2}=k_{2 y}^{2}-K_{2}^{2}$, so that the first term corresponds (of course) to the previous result for the 1-loop Bhabha maxcut, where the momentum transfer $t$ is spacelike.

The calculation of the maxcut continues with the (almost trivial) integration in $k_{20}, k_{2 y}$ and $K_{2}$ of the 3 remaining $\delta$ functions, until a last integration in $k_{2 x}$ is left. That last integration requires only some care in working out the integration ranges which satisfy the positivity constraints. Apart from an overall common factor (depending on $d$, but irrelevant), the result, say $B_{7}(d, s, t)$ can be written as

$$
B_{7}(d, s, t)=\frac{1}{\sin \left((d-3) \frac{\pi}{2}\right)} F_{1}(d, s, t)+F_{2}(d, s, t),
$$

where the two functions $F_{i}(d, s, t)$ have the form of a definite integral over a single variable of suitable expressions. Skipping any detail, the two integrals, expanded in powers of (d-4) up to next to leading order, read

$$
\begin{aligned}
& F_{1}(d, s, t)=\frac{1}{s\left(s-4 m^{2}\right) t}\left[\frac{1}{d-4}+\ln \left(s-4 m^{2}\right)-\ln \left(s+t-4 m^{2}\right)+\ln (-t)-2 \ln (2)\right], \\
& F_{2}(d, s, t)=\frac{1}{s\left(s-4 m^{2}\right) t}\left[\frac{1}{d-4}+\ln (-t)-2 \ln (2)\right] .
\end{aligned}
$$

The two terms are different; let us discuss that difference.

By standard methods, one finds that the scalar amplitude corresponding to the double box Bhabha graphs of Fig.(2) satisfies a second order differential equation in $s$ or $t$. The homogeneous part of the equation in $t$ (marginally simpler than the equation in $s$ ) reads [2]

$$
\begin{array}{r}
t\left(s+t-4 m^{2}\right) \frac{d^{2}}{d t^{2}} B(d, s, t) \\
+\left[3 t+2\left(s-4 m^{2}\right)-\frac{1}{2}\left(s-4 m^{2}\right)(d-4)\right] \frac{d}{d t} B(d, s, t) \\
+\left[1+\frac{s-4 m^{2}}{2 t}\left((d-4)-(d-4)^{2}\right)\right] B(d, s, t)=0 .
\end{array}
$$

According to the general discussion on the properties of the maxcuts, $B_{7}(d, s, t)$, Eq.(3.3), must satisfy Eq.(3.5) - and that is indeed the case, at leading and next-to-leading order in $(d-4)$, as can be easily verified by an explicitly calculation.

But it turns out that the equation is satisfied (always up to next-to-leading order in $(d-4)$ included, i.e. up to zeroth order) also by the two terms $F_{1}(d, s, t)$ and $F_{2}(d, s, t)$ taken separately (and the same applies to the $s$ equation, not discussed here). In particular, the coefficient, say $C_{1}(s, t)$, of the leading order in $(d-4)$ of the two solution, i.e. the the polar singularity $1 /(d-4)$, which is the same for both the solutions

$$
C_{1}(s, t)=\frac{1}{s\left(s-4 m^{2}\right) t},
$$

satisfies the leading order in $(d-4)$ of Eq.(3.5), which reads

$$
\left[t\left(s+t-4 m^{2}\right) \frac{d^{2}}{d t^{2}}+\left[3 t+2\left(s-4 m^{2}\right)\right] \frac{d}{d t}+1\right] C(s, t)=0 .
$$


On the other hand, the difference, of $F_{1}(d, s, t)$ and $F_{2}(d, s, t)$, which are both solutions of the linear equation Eq.(3.5) up to zeroth order in $(d-4)$, namely

$$
F_{1}(d, s, t)-F_{2}(d, s, t)=\frac{1}{s\left(s-4 m^{2}\right) t}\left[\ln \left(s-4 m^{2}\right)-\ln \left(s+t-4 m^{2}\right)\right],
$$

is also a solution of Eq.(3.6) up to zeroth order in $(d-4)$. Let us call $C_{2}(s, t)$ that difference,

$$
C_{2}(s, t) \frac{1}{s\left(s-4 m^{2}\right) t}\left[\ln \left(s-4 m^{2}\right)-\ln \left(s+t-4 m^{2}\right)\right],
$$

which is of zeroth order in $(d-4)$; it turns out that $C_{2}(s, t)$ is also a solution of Eq.(3.6), besides $C_{1}(s, t)$.

We obtain in that way, from the direct calculation of the maxcut $B_{7}(d, s, t)$, the two independent solutions of the leading term in $(d-4)$ of the homogeneous part of the second order equation in $t$ for the Bhabha scalar amplitude (and the same applies to to the equation in $s$ as well).

\section{The subgraphs}

We consider now the maxcuts of the subtopologies of the double box planar Bhabha graph in which some of the electron propagators is missing,

By removing from $\operatorname{Fig}(2)$ the electron propagator of momentum $p_{1}+k_{2}$ one obtains the graph of $\operatorname{Fig}(3)$ :

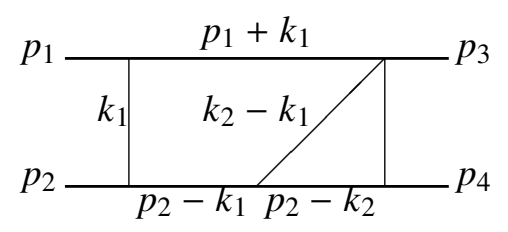

Figure 3: A 6-propagator subgraph.

The graph has 6 propagators, hence the integrand of the maxcut is the product of $6 \delta$ functions, to be integrated on the same 9 integration variables of the double box involving $7 \delta$ 's, so that the $\delta$ 's leave unconstrained one more integration variable with respect to the previous case, and therefore apparently one more non trivial integration. As the $k_{2}$ loop, however, is entirely internal to the vertex subgraph of Fig.(3) (corresponding to the process $\left.\left(p_{2}-k_{1}\right) \rightarrow\left(p_{3}-p_{1}-k_{1}\right)+p_{4}\right)$, it turns out that it is more convenient to parametrise the $k_{2}$ loop with 5 integration variables and the $k_{1}$ loop with 4 (contrary to the double box case, where the $k_{1}$ loop was given 5 integration variables), and to integrate all the $k_{2}$ variables loop first. The resulting vertex amplitude (dropping an overall angular factor) can be written as

$$
\begin{aligned}
V\left(V 1, V_{2}, V_{3}\right) & =-\frac{1}{2} \frac{\pi}{d-4} \frac{\cos \left(\pi \frac{d-4}{2}\right)}{\sin \left(\pi \frac{d-4}{2}\right)} \times \Theta\left(R_{2}\left(-m^{2}, V_{2}, V_{3}\right)\right) \\
& \times m^{d-4}\left(V_{2}^{2}\right)^{\frac{d-4}{2}}\left(R_{2}\left(-m^{2}, V_{2}, V_{3}\right)\right)^{-\frac{1}{2}+\frac{d-4}{2}}
\end{aligned}
$$


where the arguments of $V$ are $\quad V_{1}=\left(p_{2}-k_{1}\right)^{2}, \quad V_{2}=\left(p_{3}-p_{1}-k_{1}\right)^{2}, \quad V_{3}=p_{4}^{2}=-m^{2}$, and $R_{2}\left(V_{1}, V_{2}, V_{3}\right)=V_{1}^{2}+V_{2}^{2}+V_{3}^{2}-2 V_{1} V_{2}-2 V_{2} V_{3}-2 V_{1} V_{3}$.

The completion of the calculation of the maxcut with the integration in the four variables related to $k_{1}$ is then rather simple; dropping again an overall factor, the result for the maxcut of the 6-propagator Bhabha subtopology, including leading and next to leading terms in $(d-4)$ is

$$
\begin{aligned}
B_{6}(d, s, t)= & \frac{1}{\sqrt{s\left(s-4 m^{2}\right)} \sqrt{t\left(t-4 m^{2}\right)}} \\
\times & {\left[-\frac{1}{d-4}\right.} \\
& \left.\left.+\ln (2 m)+\frac{1}{2} \ln \frac{s+t-4 m^{2}}{s-4 m^{2}}-\ln (-t)-\frac{1}{2} \ln \left(4 m^{2}-t\right)\right] .\right)
\end{aligned}
$$

It is easy to verify, as in the case of the maxcut of the planar double box graph, that $B_{6}(d, s, t)$ satisfies the homogeneous equations for the amplitude of Fig.(3).

The next subgraph to consider is the 5 propagator graph of Fig.(4), obtained by removing the electron propagator of momentum $p_{2}-k_{1}$ from the graph of Fig.(3). The maxcut of its scalar

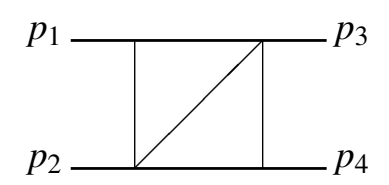

Figure 4: A 5-propagator subgraph.

amplitude consists of the same vertex subgraph appearing in Fig.(3) and given by Eq.(4.1), times two $\delta$ functions only, to be integrated on the 4 variables of the $k_{1}$ loop, amounting to the evaluation of a non trivial double integral. That is not surprising, as the graph of Fig.(4) has 5 Master Integrals, so that the scalar amplitude satisfies a 5th order homogeneous differential equation in $s$ or $t$.

The work on the amplitude is still in progress.

\section{Conclusions}

The direct calculation of maximally cut (maxcut) amplitudes can provide with the very first step in the evaluation of Feynman graphs when the differential equation approach is used.

However, when the components of the loop momenta, within the standard $d$ continuous regularisation, are parametrised in the standard way (one Minkoskian component only, while the other phyisical and regularsing components are all Euclidean) the maxcut of most graphs vanish, as the integration variables are overconstrained by the $\delta$ functions, whose arguments become positive definite (and therefore never vanishing).

As recalled in the first Section, in the $d$ continuous regularisation approach the regularising components of the loop momenta do not span the space directions spanned by the physical vectors, and therefore can be Wick-rotated (or, better, counterrotated) whitout changing the value of the considered Feynman amplitude. As any Feynman propagator is the sum of two terms, a Principal Value and a $\delta$ function, an N-propagator graph is in fact the sum of $2^{N}$ terms, one of them (the term 
involving $\delta$ functions only) being the maxcut. When the Wick-counterotation of the regularising components is performed, even if the sum of the $2^{N}$ terms remains unchanged, the value of each of the terms can change; in the maxcut, in particular, the positivity constraints on the arguments of the $\delta$ 's are relaxed, due to the change of the metric for the regularising components - and the maxcut no longer vanishes.

\section{Acknowledgements}

The author is grateful to J. Vermaseren for his assistance in the use of the algebraic program FORM [3] which was intensively used in all the steps of this work and to L. Mattiazzi for providing him with the explicit formulas of several equations.

\section{References}

[1] J.M. Henn and V.A.Smirnov, "Analytic results for two-loop master integrals for Bhabha scattering I" , JHEP 1311 (2013) 041.

[2] Courtesy of Luca Mattiazzi.

[3] J. A. M. Vermaseren, "New features of FORM," math-ph/0010025. 